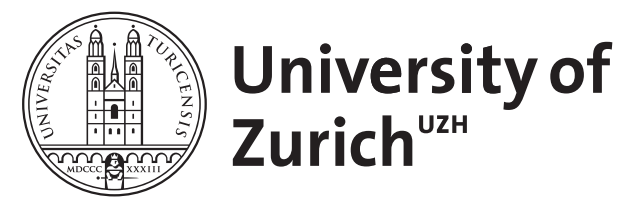

\title{
Destackification with restricted root operations
}

\author{
Kresch, Andrew
}

\begin{abstract}
Bergh's functorial destackification algorithm which employs mth root operations only for positive integers $m$ not divisible by a given prime $p$ when applied to stacks whose geometric stabilizer group schemes do not contain pth roots of unity. When $p$ is the characteristic of a given base field, this has the consequence that it is possible to destackify a tame Deligne-Mumford stack over the base field staying entirely within the realm of tame Deligne-Mumford stacks, rather than requiring more general tame Artin stacks. The modifications are based on two observations. First, destackification is possible for stacks with 3-torsion diagonalizable stabilizers, as for 2-torsion stabilizers, with just ordinary blow-ups, and not the more general stacky blow-ups that enlarge stabilizer groups. Second, modulo a given prime greater than or equal to 5, every nonzero residue class can be expressed as a product of smaller primes different from $\mathrm{p}$.
\end{abstract}

DOI: https://doi.org/10.1007/s40879-018-0281-6

Posted at the Zurich Open Repository and Archive, University of Zurich

ZORA URL: https://doi.org/10.5167/uzh-157648

Journal Article

Accepted Version

Originally published at:

Kresch, Andrew (2018). Destackification with restricted root operations. European Journal of Mathematics, 4(4):1421-1432.

DOI: https://doi.org/10.1007/s40879-018-0281-6 


\title{
DESTACKIFICATION WITH RESTRICTED ROOT OPERATIONS
}

\author{
ANDREW KRESCH
}

\begin{abstract}
We exhibit a modified version of Bergh's functorial destackification algorithm which employs $m$ th root operations only for positive integers $m$ not divisible by a given prime $p$ when applied to stacks whose geometric stabilizer group schemes do not contain $p$ th roots of unity. When $p$ is the characteristic of a given base field, this has the consequence that it is possible to destackify a tame Deligne-Mumford stack over the base field staying entirely within the realm of tame Deligne-Mumford stacks, rather than requiring more general tame Artin stacks. The modifications are based on two observations. First, destackification is possible for stacks with 3-torsion diagonalizable stabilizers, as for 2-torsion stabilizers, with just ordinary blow-ups, and not the more general stacky blowups that enlarge stabilizer groups. Second, modulo a given prime greater than or equal to 5 , every nonzero residue class can be expressed as a product of smaller primes different from $p$.
\end{abstract}

\section{Introduction}

Let $k$ be a field and $X$ a smooth separated Deligne-Mumford stack of finite type over $k$. We suppose that $X$ is tame, meaning that the characteristic of $k$ does not divide the order of the stabilizer group at any geometric point of $X$, or equivalently, $X$ has the étale local structure over its coarse moduli space of a quotient stack $[U / \Gamma]$ where $U$ is a smooth affine $k$-scheme and $\Gamma$ is a finite group whose order is not divisible by the characteristic of $k$ [3]. Bergh's functorial destackification [4] provides a mechanism to replace $X$, through a sequence of elementary operations, by a smooth stack $\widetilde{X}$ proper over $X$, such that the coarse moduli space of $\widetilde{X}$ is smooth and $\widetilde{X}$ may be recovered from its coarse moduli space by adding stabilizers in a controlled way over a simple normal crossing divisor. When $k$ has characteristic $p>0$ it is possible that stabilizer group schemes $\mu_{m}$ ( $m$ th roots of unity) with $p$ dividing $m$ arise, and $\widetilde{X}$ no longer has the simple local form $[U / \Gamma]$ but rather belongs to the more general class of tame Artin stacks [2].

In this note we provide simple modifications to the pioneering construction of Bergh that yield $\widetilde{X}$, as above, staying within the class of tame Deligne-Mumford stacks. From many points of view, (tame) Deligne-Mumford stacks are technically simpler to work with than (tame) Artin stacks.

The operations involved in Bergh's destackification are of two kinds: (i) blowing up along a smooth center, and (ii) root stack along a smooth divisor. A root operation has an attached positive integer $m$ and the effect, along a given divisor, of replacing each stabilizer group by a $\mu_{m}$-extension, without altering the coarse moduli space.

That root operations are essential for destackification is explained in [4, Example 4.3] with an example of the form $\left[\mathbb{A}^{2} / \mu_{5}\right]$. An elementary observation, stated in $[10]$,

Date: August 9, 2018.

2010 Mathematics Subject Classification. 14A20 (primary); 14E15 (secondary). 
is that when every geometric point of $X$ has stabilizer $\left(\mu_{2}\right)^{n}$ for some $n \geq 0$, Bergh's destackification algorithm yields a succession of blow-ups without nontrivial root operations. With the modifications given here, stabilizers $\left(\mu_{3}\right)^{n}$ are also destackified by successions of blow-ups without nontrivial root operations. Stabilizers $\left(\mu_{3}\right)^{n}$ and destackification have appeared in the context of Brauer-Severi surface bundles [9].

After some recollection about Bergh's destackification in Section 2 we propose modifications in Section 3. The main theorem is stated and proved in Section 4.

Acknowledgments. The author would like to thank David Rydh for stimulating discussions and helpful comments, and the referee for careful reading and valuable suggestions.

\section{BERGH'S DESTACKIFICATION}

Let $S$ be a quasi-compact scheme. The basic data, called a standard pair in [4], consist of a tame Artin stack $X$, smooth and of finite presentation over $S$, and a finite ordered set $\boldsymbol{E}=\left(E^{1}, \ldots, E^{r}\right)$ of effective Cartier divisors on $X$, smooth over $S$, which when taken together form a simple normal crossing divisor.

Associated with a standard pair is a $\mathbb{G}_{m}^{r}$-torsor $U \rightarrow X$. When $U$ is a gerbe over its coarse moduli space, we call $(X, \boldsymbol{E})$ divisorial (cf. [4, Rmk. 7.14]). A divisorialification algorithm is presented in [4] when $X$ has diagonalizable stabilizers and in [5] in general. Divisorialification produces a standard pair in which the stack is a gerbe over a tame Artin stack with diagonalizable stabilizers.

The divisorialification algorithm requires only the operation of blowing up. The center of blow-up, here and below, always is smooth over $S$ and has simple normal crossings with $\boldsymbol{E}$. After each blow-up, $\boldsymbol{E}$ is replaced by the strict transforms of its elements together with the exceptional divisor, labelled as $E^{r+1}$.

The destackification algorithm requires as well the $m$ th root stack operation for positive integers $m[7][1]$, along a divisor $E^{i}$.

In the destackification algorithm, a further structure known as a distinguished structure $\boldsymbol{D} \subset \boldsymbol{E}$ of the form $\boldsymbol{D}=\left(E^{d+1}, \ldots, E^{r}\right)$ for some $0 \leq d \leq r$ is carried along. A blow-up is called admissible when its center is contained in $E^{d+1} \cup \cdots \cup E^{r}$; a root operation along $E^{i}$ is admissible when $i \in\{d+1, \ldots, r\}$. After admissible blowup, when we replace $\boldsymbol{E}$ as described above, we have a new distinguished structure $\boldsymbol{D}=\left(E^{d+1}, \ldots, E^{r+1}\right)$.

At the heart of the destackification algorithm is a partial toric destackification algorithm of a combinatorial nature. Here the essential combinatorial data take the form of an orbifold fan consisting of a lattice $N$, a simplicial fan $\Sigma$ whose support spans $N_{\mathbb{R}}$, and a homomorphism $\beta$ to $N$ from the free abelian group with basis the set $\Sigma(1)$ of rays in $\Sigma$, sending the generator corresponding to a given ray to a nonzero lattice point on the ray. We recall, to be a simplicial fan means that every cone is simplicial, i.e., is the span of a collection of linearly independent elements of $N$. The toric stack construction [6] [8] associates to such combinatorial data an algebraic stack $X_{(N, \Sigma, \beta)}$, smooth over $S$, with normal crossing divisor whose irreducible components are in bijective correspondence with $\Sigma(1)$. This is upgraded to a standard pair by numbering the rays of $\Sigma$. Then, to give a distinguished structure means that some of the rays are identified as distinguished; specifically, for some $d \in \mathbb{N}$ the distinguished rays are the ones indexed by integers larger than $d$. Given a ray $\langle v\rangle \in \Sigma(1)$, where $v$ is a primitive lattice element and $m$ is the positive integer with $\beta(\langle v\rangle)=m v$, the corresponding divisor has generic stabilizer group $\mu_{m}$. The stack $X_{(N, \Sigma, \beta)}$ may be 
obtained from a smooth scheme over $S$ via the iterated root stack construction $[7$, Def. 2.2.4] if and only if every cone of $\Sigma$ is spanned by part of a $\mathbb{Z}$-basis of $N$.

Let $\sigma \in \Sigma$ be a cone, and let $N_{\sigma}$ be the $\mathbb{Z}$-span of $\sigma \cap N$. So $N_{\sigma}$ is saturated, hence is a direct summand of $N$. The $\mathbb{Z}$-span of the lattice points on the rays of $\sigma$ is a finite-index subgroup which we denote by $N_{\sigma}^{1}$. We have $N_{\sigma}^{1}=N_{\sigma}$ if and only if $\sigma$ is spanned by part of a $\mathbb{Z}$-basis of $N$. If $\tau$ is a face of $\sigma$, then $N_{\tau}$ is a direct summand of $N_{\sigma}$, and $N_{\tau}^{1}$ is a direct summand of $N_{\sigma}^{1}$. We have $N_{\tau}^{1}=N_{\tau} \cap N_{\sigma}^{1}$, and hence the vertical homomorphisms in the commutative diagram

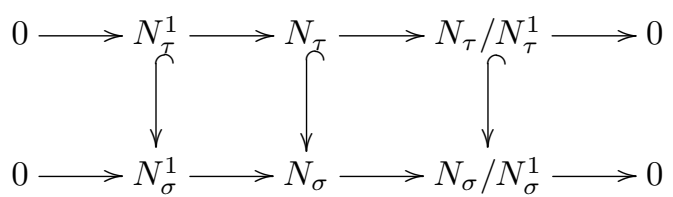

are injective.

The associated polytope $P_{\sigma}$, consisting of all real linear combinations of primitive lattice points on the rays of $\sigma$ with coefficients in the half-open interval $[0,1)$, can be identified with $\left(N_{\sigma}\right)_{\mathbb{R}} / N_{\sigma}^{1}$. The lattice points in $P_{\sigma}$ are thus in bijective correspondence with $N_{\sigma} / N_{\sigma}^{1}$, whose order is therefore the multiplicity of $\sigma$. Given a class $\bar{\psi} \in N_{\sigma} / N_{\sigma}^{1}$ we call the unique lift $\psi \in N_{\sigma} \cap P_{\sigma}$ the canonical representative.

Suppose $\tau \subsetneq \sigma=\langle\tau, v\rangle$ are cones in $\Sigma$. The ray $\langle v\rangle$ is said to be independent at $\sigma$ if the injective homomorphism $N_{\tau} / N_{\tau}^{1} \rightarrow N_{\sigma} / N_{\sigma}^{1}$ is an isomorphism, and is called independent if it is independent at $\sigma$ for all $\sigma \in \Sigma$ with $v \in \sigma$. The partial toric destackification algorithm produces an orbifold fan in which all distinguished rays are independent.

Lemma 2.1. Let $(N, \Sigma, \beta)$ be an orbifold fan, whose rays are numbered and endowed with a distinguished structure. Then the following are equivalent.

(i) All distinguished rays are independent.

(ii) For every cone $\rho \in \Sigma$, with face $\rho^{\text {nd }}$ generated by the non-distinguished rays of $\rho$, the homomorphism $N_{\rho^{\text {nd }}} / N_{\rho^{\text {nd }}}^{1} \rightarrow N_{\rho} / N_{\rho}^{1}$ is an isomorphism.

Proof. The homomorphism in (ii) factors as a composite of injective homomorphisms $N_{\tau} / N_{\tau}^{1} \rightarrow N_{\sigma} / N_{\sigma}^{1}$ as in the definition of independent ray, for cones $\sigma=\langle\tau, v\rangle$ with $\langle v\rangle$ distinguished. From this observation the equivalence is clear.

Definition 2.2. Given a cone $\rho \in \Sigma$ we denote by

$$
i_{\rho}: N_{\rho^{\mathrm{nd}}} / N_{\rho^{\mathrm{nd}}}^{1} \rightarrow N_{\rho} / N_{\rho}^{1}
$$

the homomorphism from statement (ii) of Lemma 2.1 and say that $\rho$ satisfies the independence condition if the condition in (ii) for $\rho$ is satisfied.

So, all distinguished rays of $\Sigma$ are independent if and only if every cone of $\Sigma$ satisfies the independence condition.

Lemma 2.3. Let $(N, \Sigma, \beta)$ be an orbifold fan, whose rays are numbered and endowed with a distinguished structure. For $\sigma, \tau \in \Sigma$ with $\tau \subset \sigma$, there is a commutative 
diagram with injective vertical maps

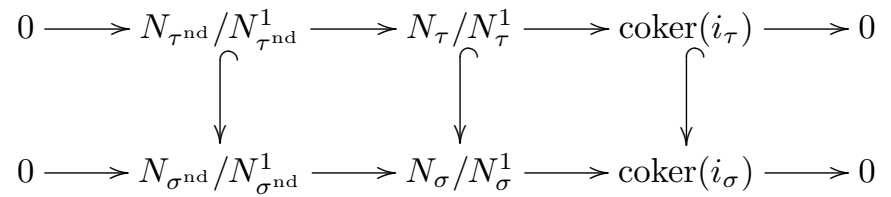

Proof. An element $\bar{\psi} \in N_{\sigma} / N_{\sigma}^{1}$ lies in the image of $N_{\tau} / N_{\tau}^{1} \rightarrow N_{\sigma} / N_{\sigma}^{1}$ if and only if the canonical representative $\psi \in N_{\sigma} \cap P_{\sigma}$ lies in $N_{\tau}$ (equivalently, in $N_{\tau} \cap P_{\tau}$ ). We have $\sigma^{\text {nd }} \cap \tau=\tau^{\text {nd }}$. So, it is clear that the intersection of the images of $N_{\tau} / N_{\tau}^{1}$ and $N_{\sigma^{\text {nd }}} / N_{\sigma^{\text {nd }}}^{1}$ in $N_{\sigma} / N_{\sigma}^{1}$ is equal to the image of $N_{\tau^{\text {nd }}} / N_{\tau^{\text {nd }}}^{1}$.

Corollary 2.4. If $\sigma \in \Sigma$ satisfies the independence condition, then so does every face of $\sigma$.

Definition 2.5. The distinguished multiplicity of a cone $\sigma \in \Sigma$ is the order of $\operatorname{coker}\left(i_{\sigma}\right)$. We denote this quantity by $\operatorname{dmult}(\sigma)$.

We have

$$
\operatorname{mult}(\sigma)=\operatorname{dmult}(\sigma) \cdot \operatorname{mult}\left(\sigma^{\mathrm{nd}}\right) .
$$

By Lemma 2.3 the distinguished multiplicity of any face of $\sigma$ divides $\operatorname{dmult}(\sigma)$.

In the partial toric destackification algorithm the blow-ups always have, as center, an intersection of components of the normal crossing divisor, corresponding in terms of combinatorial data to an element $\sigma \in \Sigma$. If $I \subset \Sigma(1)$ denotes the set of rays of $\sigma$, then the center of blow-up is $\bigcap_{i \in I} E^{i}$. After blowing up, the exceptional divisor $E^{r+1}$ corresponds to $\left\langle v_{r+1}\right\rangle$, where

$$
v_{r+1}=\sum_{i \in I} \beta\left(\left\langle v_{i}\right\rangle\right)
$$

and, in a process known as star subdivision, the cone $\sigma$ is replaced by $|I|$ new cones of the same dimension, each spanned by $|I|-1$ of the rays of $\sigma$ and $v_{r+1}$. The homomorphism $\beta$ is extended by $\left\langle v_{r+1}\right\rangle \mapsto v_{r+1}$. The other kind of operation, $m$ th root along $E^{i}$, leaves the fan unchanged and multiplies the value of $\beta\left(\left\langle v_{i}\right\rangle\right)$ by $m$.

The partial toric destackification algorithm can be summarized as follows. A cone $\sigma \in \Sigma$ which does not satisfy the independence condition is chosen, as well as a class $\bar{\psi} \in N_{\sigma} / N_{\sigma}^{1}$, not in the image of $i_{\sigma}$, with canonical representative $\psi \in N_{\sigma} \cap P_{\sigma}$. Let $c$ denote the order of $\bar{\psi}$. Now $\psi$ lies in the interior of $P_{\tau}$ in $\left(N_{\tau}\right)_{\mathbb{R}}$ for a unique face $\tau$ of $\sigma$. We denote by $I$ the indices of the non-distinguished rays of $\tau$ and by $I^{\prime}$ the indices of the distinguished rays; we have $I^{\prime} \neq \emptyset$, and for $i \in I \cup I^{\prime}$ write $v_{i}$ for the primitive lattice element that generates the $i$ th ray. Then

$$
\sum_{i \in I} a_{i} v_{i}+\sum_{i \in I^{\prime}} b_{i} v_{i}=c \psi
$$

for some positive integer coefficients $a_{i}, b_{i}$, such that $a_{i}, b_{i}$, and $c$ have no common prime factor. We define positive integers $m_{i}$ by $\beta\left(\left\langle v_{i}\right\rangle\right)=m_{i} v_{i}$ and write, analogously,

$$
\sum_{i \in I} A_{i} m_{i} v_{i}+\sum_{i \in I^{\prime}} B_{i} m_{i} v_{i}=C \psi,
$$

for some positive integer coefficients $A_{i}, B_{i}$, and $C$ without common prime factor. We observe, by the definition of canonical representative, that $a_{i}<c$ for all $i \in I$ 
and $b_{i}<c$ for all $i \in I^{\prime}$. Now we apply the $B_{i}$ th root construction to the divisor corresponding to $\left\langle v_{i}\right\rangle$ for all $i \in I^{\prime}$ and star subdivision to the cone $\tau$ :

$$
\sum_{i \in I_{1}}\left(A_{i}-1\right) m_{i} v_{i}+\left(\sum_{i \in I} m_{i} v_{i}+\sum_{i \in I^{\prime}} B_{i} m_{i} v_{i}\right)=C \psi
$$

where $I_{1}=\left\{i \in I \mid A_{i}>1\right\}$. If $I_{1} \neq \emptyset$ then another star subdivision is performed, with the cone spanned by $v_{i}$ for $i \in I_{1}$ and $\sum_{i \in I} m_{i} v_{i}+\sum_{i \in I^{\prime}} B_{i} m_{i} v_{i}$. This is continued until the fan contains the ray $\langle\psi\rangle$.

Lemma 2.6. Let $(N, \Sigma, \beta)$ be an orbifold fan, whose rays are numbered and endowed with a distinguished structure. Let $\tau \in \Sigma$ and $\bar{\psi} \in N_{\tau} / N_{\tau}^{1}$ be given, with $\bar{\psi}$ not in the image of $i_{\tau}$, along with a representative $\psi \in N_{\tau}$, satisfying relation (1) for some positive integer coefficients $a_{i}, b_{i}$, and $c$, where $I$ indexes the non-distinguished rays and $I^{\prime}$ indexes the distinguished rays of $\tau$. We let $\left(N, \Sigma^{\prime}, \beta^{\prime}\right)$ be the outcome of the procedure described above, consisting of an initial root operation and repeated star subdivisions to obtain $\langle\psi\rangle \in \Sigma^{\prime}$. Then, for every $\sigma \in \Sigma$ having $\tau$ as a face and $\sigma^{\prime} \in \Sigma^{\prime}$ of the same dimension as and contained in $\sigma$, the number of distinguished rays of $\sigma^{\prime}$ is greater than or equal to the number of distinguished rays of $\sigma$, and in case of equality we have

$$
\frac{\operatorname{dmult}\left(\sigma^{\prime}\right)}{\operatorname{dmult}(\sigma)}=\frac{\operatorname{mult}\left(\sigma^{\prime}\right)}{\operatorname{mult}(\sigma)} \in\left\{\frac{b_{i}}{c d} \mid i \in I^{\prime}, d \in \mathbb{N}_{>0}\right\}
$$

Proof. The hypotheses imply $N_{\sigma^{\prime}}=N_{\sigma}$. Since the new ray added by a star subdivision is distinguished, $\sigma^{\prime}$ must have at least as many distinguished rays as $\sigma$. We suppose from now on that they have the same number of distinguished rays. Then $\sigma^{\prime \text { nd }}=\sigma^{\text {nd }}$, which implies the claimed equality of ratios. Let us write

$$
\psi=d \psi_{0}
$$

with $d \in \mathbb{N}_{>0}$ and $\psi_{0}$ a primitive element of $N_{\tau}$. For some $i^{\prime} \in I^{\prime}$ we have $\sigma^{\prime}$ generated by $\psi_{0}$ and the rays of $\sigma$ except $\left\langle v_{i^{\prime}}\right\rangle$. So $N_{\sigma^{\prime}}^{1}$ is the $\mathbb{Z}$-span of $\psi_{0}$ and the lattice points on the rays of $\sigma$ except $\left\langle v_{i^{\prime}}\right\rangle$. Let $\Gamma$ be the $\mathbb{Z}$-span of $b_{i^{\prime}} v_{i^{\prime}}$ and the lattice points on the rays of $\sigma$ except $\left\langle v_{i^{\prime}}\right\rangle$. Then $\Gamma$ is a subgroup of $N_{\sigma}^{1}$ of index $b_{i^{\prime}}$ and, as well, a subgroup of $N_{\sigma^{\prime}}^{1}$ of index $c d$. It follows that $\operatorname{mult}\left(\sigma^{\prime}\right) / \operatorname{mult}(\sigma)=b_{i^{\prime}} / c d$.

The precise algorithm stipulates all choices above unambiguously, as will now be described. We order pairs $(n, d) \in \mathbb{N}^{2}$ with $d>1$ lexicographically and consider

$\{($ number of nondistinguished rays, $\operatorname{dmult}(\sigma)) \mid$ $\sigma \in \Sigma$ not satisfying the independence condition $\}$.

If the set is empty, then by Lemma 2.1, all distinguished rays are independent. Otherwise we let $\sigma \in \Sigma$ be a cone for which the pair attains the maximum value; when there is more than one such cone the lexicographically largest one is taken, where the cones are labelled by listing the indices of their rays in ascending order. Let $\bar{\psi} \in N_{\sigma} / N_{\sigma}^{1}$ be an element, not in the image of $i_{\sigma}$, with canonical representative $\psi \in N_{\sigma} \cap P_{\sigma}$. The minimal such $\psi$ is taken, under the description of $\psi$ as a rational linear combination of the rays of $\sigma$, with tuples of rational numbers ordered lexicographically. We let $\tau$ denote the face of $\sigma$ with the property that $\psi$ lies in the interior of $P_{\tau}$. Then $a_{i}, b_{i}$, and $c$ are defined as in (1) and the corresponding sequence of operations is performed to obtain $\left(N, \Sigma^{\prime}, \beta^{\prime}\right)$. 
Proposition 2.7. Let $(N, \Sigma, \beta)$ be an orbifold fan, whose rays are numbered and endowed with a distinguished strucuture. We suppose that not all distinguished rays are independent, and follow the procedure described above to obtain $\left(N, \Sigma^{\prime}, \beta^{\prime}\right)$. Let us attach to $(N, \Sigma, \beta)$ the sequence of pairs of integers

$$
\text { (number of nondistinguished rays, } \operatorname{dmult}(\sigma) \text { ) }
$$

among all $\sigma \in \Sigma$ not satisfying the independence condition, sorted into weakly descending order under the lexicographic ordering of pairs. Then the corresponding sequence attached to $\left(N, \Sigma^{\prime}, \beta^{\prime}\right)$ is a lexicographically smaller sequence of pairs of integers.

Proof. Let $(n, d)$ denote the maximum value, among pairs, and $\sigma \in \Sigma$ the chosen cone. We claim:

(i) Any cone $\rho^{\prime} \in \Sigma^{\prime} \backslash \Sigma$ has invariant $\left(n^{\prime}, d^{\prime}\right)$ that is lexicographically smaller than $(n, d)$; here, $n^{\prime}$ denotes the number of nondistinguished rays of $\rho^{\prime}$, and $d^{\prime}$, the distinguished multiplicity.

(ii) We have $\sigma \notin \Sigma^{\prime}$.

These assertions clearly imply the proposition.

If $\left(N, \widetilde{\Sigma}^{\prime}, \tilde{\beta}^{\prime}\right)$ is the result of star subdivision of a cone $\tilde{\tau} \in \widetilde{\Sigma}$ of an orbifold fan $(N, \widetilde{\Sigma}, \tilde{\beta})$, then each cone $\tilde{\rho}^{\prime} \in \widetilde{\Sigma}^{\prime} \backslash \widetilde{\Sigma}$ is a face of some $\tilde{\sigma}^{\prime} \in \widetilde{\Sigma}^{\prime}$, with $\tilde{\sigma}^{\prime}$ of the same dimension as and contained in some $\tilde{\sigma} \in \widetilde{\Sigma}$ having $\tilde{\tau}$ as a face. It follows that for $\rho^{\prime} \in \Sigma^{\prime} \backslash \Sigma$, there exists $\sigma^{\prime} \in \Sigma^{\prime}$ having $\rho^{\prime}$ as a face, where $\sigma^{\prime}$ has the same dimension as and is contained in some cone of $\Sigma$ that has $\tau$ as a face.

If $\rho^{\prime} \in \Sigma^{\prime} \backslash \Sigma$, then there exist $\eta^{\prime} \in \Sigma^{\prime}$ having $\rho^{\prime}$ as a face and $\eta \in \Sigma$ having $\tau$ as a face, such that $\eta^{\prime}$ has the same dimension as and is contained in $\eta$. By Lemma 2.6 , the number of nondistinguished rays of $\eta^{\prime}$ is less than or equal to the number of nondistinguished rays of $\eta$, and in case of equality, we have $\operatorname{dmult}\left(\eta^{\prime}\right)<\operatorname{dmult}(\eta)$. By Corollary 2.4, $\eta$ does not satisfy the independence condition. By the choice of $\sigma$, then, $\eta$ has at most $n$ nondistinguished rays, and in case of equality, $\operatorname{dmult}(\eta) \leq d$. If $\rho^{\prime}$ has exactly $n$ nondistinguished rays, then so do $\eta^{\prime}$ and $\eta$. By Lemma 2.3, then, $\operatorname{dmult}\left(\rho^{\prime}\right) \leq \operatorname{dmult}\left(\eta^{\prime}\right)<\operatorname{dmult}(\eta) \leq d$.

Example 2.8. In $\mathbb{Z}^{6}$ with standard basis $e_{1}, \ldots, e_{6}$ we consider the cone $\left\langle v_{1}, \ldots, v_{6}\right\rangle$ spanned by

$v_{1}=e_{1}, \quad v_{2}=e_{2}, \quad v_{3}=e_{3}, \quad v_{4}=e_{4}, \quad v_{5}=(2,1,1,0,3,0), \quad v_{6}=(1,1,0,1,0,3)$, where rays are taken in the order listed and $v_{4}, v_{5}, v_{6}$ are distinguished. This gives rise to an orbifold fan with lattice $N=\mathbb{Z}^{6}$, fan $\left\{\left\langle v_{i}\right\rangle_{i \in I} \mid I \subset\{1, \ldots, 6\}\right\}$, and homomorphism $\bigoplus_{i=1}^{6} \mathbb{Z}\left\langle v_{i}\right\rangle \rightarrow N,\left\langle v_{i}\right\rangle \mapsto v_{i}$. The cones not satisfying the independence condition are are listed in Table 1 , sorted by pair $(n, d)$ with $n$ the number of nondistinguished rays and $d$ the distinguished multiplicity (always equal to the multiplicity in this example). The canonical representative of nonzero $\bar{\psi} \in N_{\sigma} / N_{\sigma}^{1}$ is indicated for the minimal $\sigma$ with $\bar{\psi} \in N_{\sigma} / N_{\sigma}^{1}$. The largest pair $(n, d)$ is $(3,9)$, for $\sigma=\left\langle v_{1}, \ldots, v_{6}\right\rangle$, with lexicographically smallest canonical representative of a nonzero class of $N_{\sigma} / N_{\sigma}^{1}$

$$
\psi=(1,1,1,1,1,1)=0 v_{1}+\frac{1}{3} v_{2}+\frac{2}{3} v_{3}+\frac{2}{3} v_{4}+\frac{1}{3} v_{5}+\frac{1}{3} v_{6} .
$$

For the face ${ }^{1}$

$$
\tau=\left\langle v_{2}, v_{3}, v_{4}, v_{5}, v_{6}\right\rangle
$$

\footnotetext{
${ }^{1}$ Bergh's algorithm also takes into account the number of nondistinguished rays for the choice of face. We ignore this detail, which is irrelevant for correctness of algorithm, in our treatment.
} 


\begin{tabular}{lcc}
$\sigma$ & $(n, d)$ & canonical representatives $\psi \in N_{\sigma}$ \\
\hline$\left\langle v_{1}, v_{2}, v_{3}, v_{4}, v_{5}, v_{6}\right\rangle$ & $(3,9)$ & \\
$\left\langle v_{1}, v_{2}, v_{3}, v_{4}, v_{5}\right\rangle$ & $(3,3)$ & \\
$\left\langle v_{1}, v_{2}, v_{3}, v_{4}, v_{6}\right\rangle$ & $(3,3)$ & \\
$\left\langle v_{1}, v_{2}, v_{3}, v_{5}\right\rangle$ & $(3,3)$ & $(1,1,1,0,1,0),(2,1,1,0,2,0)$ \\
$\left\langle v_{1}, v_{2}, v_{3}, v_{5}, v_{6}\right\rangle$ & $(3,3)$ & \\
$\left\langle v_{1}, v_{2}, v_{4}, v_{5}, v_{6}\right\rangle$ & $(2,3)$ & \\
$\left\langle v_{1}, v_{2}, v_{4}, v_{6}\right\rangle$ & $(2,3)$ & $(1,1,0,1,0,1),(1,1,0,1,0,2)$ \\
$\left\langle v_{1}, v_{3}, v_{4}, v_{5}, v_{6}\right\rangle$ & $(2,3)$ & $(2,1,1,1,1,2),(2,1,1,1,2,1)$ \\
$\left\langle v_{2}, v_{3}, v_{4}, v_{5}, v_{6}\right\rangle$ & $(2,3)$ & $(1,1,1,1,1,1),(2,2,1,1,2,2)$
\end{tabular}

TABLE 1. Cones not satisfying the independence condition in Example 2.8. The canonical representative of a nonzero class $\bar{\psi} \in N_{\sigma} / N_{\sigma}^{1}$ is listed just once, for the smallest cone $\sigma$ with $\bar{\psi} \in N_{\sigma} / N_{\sigma}^{1}$.

the interior of $P_{\tau}$ contains $\psi$, with relation with positive integer coefficients

$$
v_{2}+2 v_{3}+2 v_{4}+v_{5}+v_{6}=3 \psi \text {. }
$$

So we root divisor 4 and star subdivide $\tau$ to obtain new ray $\langle(3,3,2,3,3,3)\rangle$. One more star subdivision yields the ray $\langle\psi\rangle$. The new largest pair $(n, d)$ is then $(3,6)$.

\section{Modifications}

The first modification to the partial toric destackification algorithm described in Section 2 is clearly compatible with correctness of algorithm.

Modification 3.1. Letting $\ell$ denote the largest prime divisor of $\operatorname{dmult}(\sigma)$, we restrict the choice of $\bar{\psi} \in N_{\sigma} / N_{\sigma}^{1}$ to $\ell$-power-torsion elements, such that the class of $\bar{\psi}$ in $\operatorname{coker}\left(i_{\sigma}\right)$ has order $\ell$.

Such $\bar{\psi}$ exists since the map from $N_{\sigma} / N_{\sigma}^{1}$ to $\operatorname{coker}\left(i_{\sigma}\right)$ is surjective on $\ell$-powertorsion subgroups. Then the positive integer $c$ in (1) is a power of $\ell$, with

$$
\frac{b_{i}}{c} \in\left\{\frac{1}{\ell}, \ldots, \frac{\ell-1}{\ell}\right\}
$$

for all $i \in I^{\prime}$.

Definition 3.2. In the situation of Modification 3.1 we suppose that $\ell=3$. Then $\bar{\psi}$ is called good if the canonical representative $\psi$ satisfies relation (1) with

$$
\frac{b_{i}}{c}=\frac{1}{3}
$$

for all $i \in I^{\prime}$. We say that $\sigma$ is good if there exists $\bar{\psi}$ as in Modification 3.1 that is good. Otherwise, we say that $\sigma$ is bad.

Modification 3.3. When the largest prime divisor of $\operatorname{dmult}(\sigma)$ is $\ell=3$, we augment the pair $(n, d)$ by an indication of badness: $(n, d, 0)$ if $\sigma$ is good; $(n, d, 1)$ if $\sigma$ is bad. If $\sigma$ is good then good $\bar{\psi}$ is taken. If $\sigma$ is bad then the algorithm is modified by replacing relation (1) by

$$
\sum_{i \in I} a_{i} v_{i}+\sum_{i \in I^{\prime}} \frac{c}{3} v_{i}=\frac{c}{3} \psi^{\prime}
$$


writing analogously

$$
\sum_{i \in I} A_{i}^{\prime} m_{i} v_{i}+\sum_{i \in I^{\prime}} B_{i}^{\prime} m_{i} v_{i}=C^{\prime} \psi^{\prime},
$$

and, with this relation, performing the corresponding sequence of root and star subdivision operations.

The analysis of Proposition 2.7 reveals, in the setting of bad $\sigma$ in Modification 3.3 , that the outcome of the star subdivision can have new cones (cones not in $\Sigma$ ) with invariant $\left(n^{\prime}, d^{\prime}\right)=(n, d)$. Indeed, the application of Lemma 2.6 in the third paragraph of the proof only yields $\operatorname{dmult}\left(\eta^{\prime}\right) \leq \operatorname{dmult}(\eta)$, with equality when $\psi^{\prime}$ is a primitive element of $N_{\tau}$. We claim that all such cones are good; the claim implies correctness of algorithm. We are quickly reduced to showing that a cone $\sigma^{\prime}$ as in Lemma 2.6 is good. With the notation of the proof of Lemma 2.6 we have two cases, according to whether $b_{i^{\prime}}$ is equal to $c / 3$ or $2 c / 3$. If $b_{i^{\prime}}=c / 3$, then the relation

$$
\psi^{\prime}+\sum_{\substack{i \in I^{\prime} \\ b_{i}=2 c / 3}} v_{i}=3 \psi
$$

confirms that $\sigma^{\prime}$ is good. Otherwise, we use the relation

$$
\sum_{i \in I} a_{i} v_{i}+\frac{c}{3} \psi^{\prime}+\sum_{\substack{i \in I^{\prime} \\ b_{i}=c / 3}} \frac{c}{3} v_{i}=c\left(2 \psi-\sum_{\substack{i \in I^{\prime} \\ b_{i}=2 c / 3}} v_{i}\right)
$$

In Example 2.8 the cone $\sigma$ is good. With Modification 3.3 the algorithm selects $\psi=(1,1,1,0,1,0)$, in the interior of $P_{\tau}$ for $\tau=\left\langle v_{1}, v_{2}, v_{3}, v_{5}\right\rangle$.

Example 3.4. We consider the same cone as in Example 2.8, but where rays $v_{3}, v_{4}$, $v_{5}, v_{6}$ are distinguished. Now the cone $\sigma$ is bad. With Modification 3.3 the algorithm selects $\psi=(1,1,1,1,1,1)$, with $\psi^{\prime}=(3,3,2,2,3,3)$ and star subdivision leading to the cones listed in Table 2 .

Modification 3.5. In comparing distinguished multiplicities, we represent a positive integer by its prime factorization, written as a string of prime numbers in weakly decreasing order, and use the lexicographic order of strings of prime numbers.

Looking at Lemma 2.6 and the constraint (2), we see that Modification 3.5 is compatible with correctness of algorithm. In fact, with Modification 3.5 the constraint (2) can be relaxed to allow any numerator whose prime factors are less than $\ell$. Thus we obtain the following modification, or rather, family of modifications, one for every collection of functions as indicated; see Section 4 for the particular choices of interest.

Modification 3.6. For every prime number $\ell \neq 3$ let $s_{\ell}:\{1, \ldots, \ell-1\} \rightarrow \mathbb{N}_{>0}$ be a function with $s_{\ell}(n) \equiv n \bmod \ell$ and all prime factors of $s_{\ell}(n)$ less than $\ell$, for all $n$. Then, when $\ell \neq 3$ we make the following modification to relation (1), where we write $c=\ell^{e}$ :

$$
\sum_{i \in I} a_{i} v_{i}+\sum_{i \in I^{\prime}} b_{i}^{\prime} v_{i}=c \psi^{\prime}, \quad b_{i}^{\prime}=\ell^{e-1} s_{\ell}\left(\frac{b_{i}}{\ell^{e-1}}\right) .
$$

We have $b_{i}^{\prime} \equiv b_{i} \bmod c$, and hence $\psi^{\prime}$ is also a representative of $\bar{\psi} \in N_{\sigma} / N_{\sigma}^{1}$. We proceed to write the analogous relation

$$
\sum_{i \in I} A_{i}^{\prime} m_{i} v_{i}+\sum_{i \in I^{\prime}} B_{i}^{\prime} m_{i} v_{i}=C^{\prime} \psi^{\prime}
$$




\begin{tabular}{lc}
$\sigma$ & $(n, d$, badness $)$ \\
\hline$\left\langle v_{1}, v_{2}, v_{3}, v_{4}, v_{5}, \psi^{\prime}\right\rangle$ & $(2,9$, good $)$ \\
$\left\langle v_{1}, v_{2}, v_{3}, v_{4}, v_{6}, \psi^{\prime}\right\rangle$ & $(2,9$, good $)$ \\
$\left\langle v_{1}, v_{2}, v_{3}, v_{5}, v_{6}, \psi^{\prime}\right\rangle$ & $(2,9$, good $)$ \\
$\left\langle v_{1}, v_{2}, v_{4}, v_{5}, v_{6}, \psi^{\prime}\right\rangle$ & $(2,9$, good $)$ \\
$\left\langle v_{1}, v_{2}, v_{3}, v_{4}, v_{5}\right\rangle$ & $(2,3$, bad $)$ \\
$\left\langle v_{1}, v_{2}, v_{3}, v_{4}, v_{6}\right\rangle$ & $(2,3$, bad $)$ \\
$\left\langle v_{1}, v_{2}, v_{3}, v_{5}\right\rangle$ & $(2,3$, bad $)$ \\
$\left\langle v_{1}, v_{2}, v_{3}, v_{5}, v_{6}\right\rangle$ & $(2,3$, bad $)$ \\
$\left\langle v_{1}, v_{2}, v_{4}, v_{5}, v_{6}\right\rangle$ & $(2,3$, bad $)$ \\
$\left\langle v_{1}, v_{2}, v_{4}, v_{6}\right\rangle$ & $(2,3$, bad $)$ \\
$\left\langle v_{1}, v_{3}, v_{4}, v_{5}, v_{6}, \psi^{\prime}\right\rangle$ & $(1,9$, good $)$ \\
$\left\langle v_{1}, v_{3}, v_{4}, v_{5}, v_{6}\right\rangle$ & $(1,3$, bad $)$ \\
$\left\langle v_{2}, v_{3}, v_{4}, v_{5}, \psi^{\prime}\right\rangle$ & $(1,3$, good $)$ \\
$\left\langle v_{2}, v_{3}, v_{4}, v_{6}, \psi^{\prime}\right\rangle$ & $(1,3$, good $)$ \\
$\left\langle v_{2}, v_{3}, v_{5}, v_{6}, \psi^{\prime}\right\rangle$ & $(1,3$, good $)$ \\
$\left\langle v_{2}, v_{4}, v_{5}, v_{6}, \psi^{\prime}\right\rangle$ & $(1,3$, good $)$ \\
$\left\langle v_{3}, v_{4}, v_{5}, v_{6}, \psi^{\prime}\right\rangle$ & $(0,3$, good $)$
\end{tabular}

TABLE 2. Cones not satisfying the independence condition in Example 3.4 after star subdivision.

and, with this relation, perform the corresponding sequence of root and star subdivision operations.

\section{MAIN THEOREM}

The main theorem asserts that for a given prime number $p$ there is a functorial destackification algorithm which, when applied to a tame Artin stack whose geometric stabilizer group schemes have no subgroup scheme isomorphic to $\mu_{p}$, performs a sequence of operations that preserve the property of having geometric stabilizer group schemes with no subgroup scheme isomorphic to $\mu_{p}$. Applied to a tame DeligneMumford stack of characteristic $p$, the destackification preserves the property of being a tame Deligne-Mumford stack of characteristic $p$.

Theorem 4.1. Let $p$ be a prime number and $S$ a quasi-compact base scheme. Then there is a destackification algorithm for standard pairs $(X, \boldsymbol{E})$ over $S$, functorial for change of base $S^{\prime} \rightarrow S$ and gerbes and smooth stabilizer preserving morphisms $X^{\prime} \rightarrow X$, with the property that if the geometric stabilizer group schemes of $X$ have no subgroup scheme isomorphic to $\mu_{p}$, then this property is maintained by the destackification.

The notion of standard pair and the operations employed in a destackification have been recalled in Section 2.

Without the property concerning geometric stabilizer group schemes containing $\mu_{p}$, this is proved in [4] (when $X$ has diagonalizable stabilizer group schemes) and [5] (in general).

Proposition 4.2. Let $p$ and $\ell$ be prime numbers with $(p, \ell) \neq(2,3)$. Then the residue classes $\bmod \ell$ of the prime numbers less than $\ell$ and different from $p$ generate $(\mathbb{Z} / \ell \mathbb{Z})^{*}$. 
Proof. We may suppose $\ell \geq 5$. An equivalent formulation of the assertion is that $(\mathbb{Z} / \ell \mathbb{Z})^{*}$ is generated by the residue classes of the element of the set $S$ of positive integers less than $\ell$, relatively prime to $p$. Since $|S| \geq(\ell-1) / 2$ we are reduced to showing that $S$ is not equal to the set positive integers less than $\ell$ that are quadratic residues $\bmod \ell$. We have $4 \notin S$ when $p=2$ and $|S|>(\ell-1) / 2$ otherwise.

Corollary 4.3. Let $p$ and $\ell$ be distinct prime numbers with $(p, \ell) \neq(2,3)$. Then the function $s_{\ell}^{(p)}:\{1, \ldots, \ell-1\} \rightarrow \mathbb{N}_{>0}$ given by

$$
s_{\ell}^{(p)}(n):=\min \{a \equiv n \bmod \ell \mid \text { all prime factors }<\ell \text { and } \neq p\},
$$

is well-defined and satisfies $s_{\ell}^{(p)}(n) \not \equiv 0 \bmod p$ for all $n \in\{1, \ldots, \ell-1\}$.

Proof of the main theorem. For the given prime $p$, the functions $s_{\ell}^{(p)}$ for $\ell \notin\{3, p\}$ from Corollary 4.3, together with $(p \neq 3) s_{p}^{(p)}(n):=n$, give an instance of Modification 3.6. This is a destackification algorithm with the desired property.

Corollary 4.4. Let $S$ be a quasi-compact base scheme and $(X, E)$ a standard pair over $S$, such that every geometric stabilizer group of $X$ is isomorphic to $\left(\mu_{3}\right)^{n}$ for some natural number $n$. Then the destackification algorithm of Theorem 4.1 yields (for any p) a succession of blow-ups without nontrivial root operations.

The analogous assertion for stabilizers $\left(\mu_{2}\right)^{n}$ is an observation made in [10].

Proof. With the notation of (1) we always have $c=3$, with $a_{i}, b_{i} \in\{1,2\}$. As well, for all $i \in I \cup I^{\prime}$ we have $m_{i}=1$. (A ray $\langle v\rangle$ of $\Sigma$ can have $\beta(\langle v\rangle)=3 v$, but then $v \in\left\{v_{i}\right\}_{i \in I \cup I}$ would lead to a stabilizer group $\mu_{9}$.) In the good case, $b_{i}=1$ for all $i \in I^{\prime}$ and the star subdivision is performed without recourse to nontrivial root operations. In the bad case, with $\psi^{\prime}=\sum_{i \in I} a_{i} v_{i}+\sum_{i \in I^{\prime}} v_{i}$ we are led to star subdivisions that do not rely on nontrivial root operations.

\section{REFERENCES}

[1] D. Abramovich, T. Graber, and A. Vistoli, Gromov-Witten theory of Deligne-Mumford stacks, Amer. J. Math. 130 (2008), 1337-1398.

[2] D. Abramovich, M. Olsson, and A. Vistoli, Tame stacks in positive characteristic, Ann. Inst. Fourier (Grenoble) 58 (2008), 1057-1091.

[3] D. Abramovich and A. Vistoli, Compactifying the space of stable maps, J. Amer. Math. Soc. 15 (2002), 27-75.

[4] D. Bergh, Functorial destackification of tame stacks with abelian stabilisers, Compositio Math. 153 (2017), 1257-1315.

[5] D. Bergh and D. Rydh, Functorial destackification and weak factorization of orbifolds, in preparation.

[6] L. A. Borisov, L. Chen, and G. G. Smith, The orbifold Chow ring of toric Deligne-Mumford stacks, J. Amer. Math. Soc. 18 (2005), 193-215.

[7] C. Cadman, Using stacks to impose tangecy conditions on curves, Amer. J. Math. 129 (2007), 405-427.

[8] I. Iwanari, Logarithmic geometry, minimal free resolutions and toric algebraic stacks, Publ. Res. Inst. Math. Sci. 45 (2009), 1095-1140.

[9] A. Kresch and Yu. Tschinkel, Models of Brauer-Severi surface bundles, preprint, 2017.

[10] J. Oesinghaus, Conic bundles and iterated root stacks, Eur. J. Math., to appear.

InStitut FÜr Mathematik, Universität ZÜrich, Winterthurerstrasse 190, CH-8057 ZÜrich, SWITZERLAND

E-mail address: andrew.kresch@math.uzh.ch 